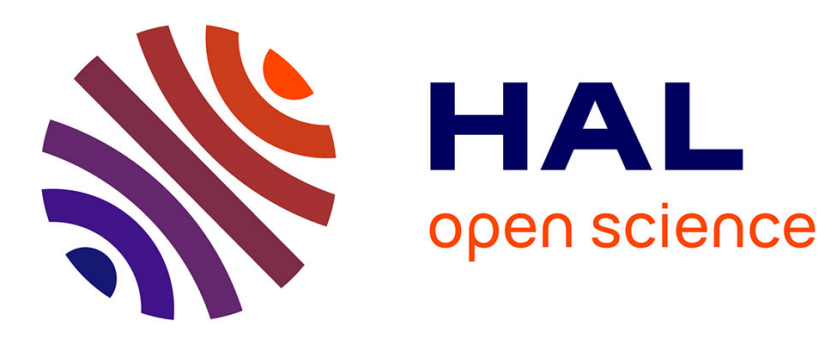

\title{
The Bounded Core for Games with Precedence Constraints
}

Michel Grabisch, Peter Sudhölter

\section{To cite this version:}

Michel Grabisch, Peter Sudhölter. The Bounded Core for Games with Precedence Constraints. 2012. halshs-00673909

\section{HAL Id: halshs-00673909 \\ https://shs.hal.science/halshs-00673909}

Submitted on 24 Feb 2012

HAL is a multi-disciplinary open access archive for the deposit and dissemination of scientific research documents, whether they are published or not. The documents may come from teaching and research institutions in France or abroad, or from public or private research centers.
L'archive ouverte pluridisciplinaire HAL, est destinée au dépôt et à la diffusion de documents scientifiques de niveau recherche, publiés ou non, émanant des établissements d'enseignement et de recherche français ou étrangers, des laboratoires publics ou privés. 


\section{Documents de Travail du Centre d'Economie de la Sorbonne}

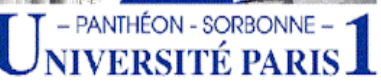

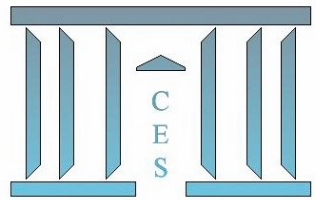

The Bounded Core for Games with Precedence Constraints

Michel GRABISCH, Peter SUDHÖLTER

2012.06

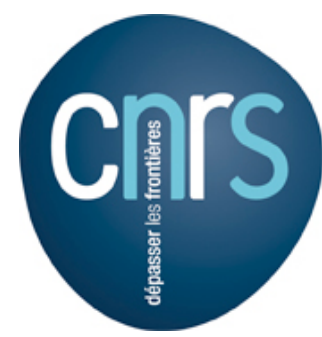

Maison des Sciences Économiques, 106-112 boulevard de L'Hôpital, 75647 Paris Cedex 13 http://centredeconomiesorbonne.univ-paris1.fr/bandeau-haut/documents-de-travail/ 


\title{
The Bounded Core for Games with Precedence Constraints*
}

\author{
Michel Grabisch ${ }^{\dagger} \quad$ Peter Sudhölter ${ }^{\ddagger}$
}

January 31, 2012

\begin{abstract}
An element of the possibly unbounded core of a cooperative game with precedence constraints belongs to its bounded core if any transfer to a player from any of her subordinates results in payoffs outside the core. The bounded core is the union of all bounded faces of the core, it is nonempty if the core is nonempty, and it is a continuous correspondence on games with coinciding precedence constraints. If the precedence constraints generate a connected hierarchy, then the core is always nonempty. It is shown that the bounded core is axiomatized similarly to the core for classical cooperative games, namely by boundedness (BOUND), nonemptiness for zero-inessential two-person games (ZIG), anonymity, covariance under strategic equivalence (COV), and certain variants of the reduced game property (RGP), the converse reduced game property (CRGP), and the reconfirmation property. The core is the maximum solution that satisfies a suitably weakened version of BOUND together with the remaining axioms. For games with connected hierarchies, the bounded core is axiomatized by BOUND, ZIG, COV, and some variants of RGP and CRGP, whereas the core is the maximum solution that satisfies the weakened version of BOUND, COV, and the variants of RGP and CRGP.
\end{abstract}

Keywords: TU game $\cdot$ Core $\cdot$ Restricted Cooperation

\section{JEL Classification: $\mathrm{C} 71$}

\section{Introduction}

In the classical theory of cooperative games one assumes that all players may cooperate, i.e., any coalition may form. However, a more general model for cooperative games with or without transferable utilities (TU or NTU) is necessary in order to describe situations in which cooperation is restricted. In this paper we adopt the model of Faigle and Kern (1992) who assume that the set of players has a hierarchical structure generated by some partial order relation. Only those coalitions may form (are feasible) that satisfy the following condition: With any player all of her subordinates (i.e., the players preceding her according to the partial order relation) must also be members of the coalition. If all players are incomparable, then any coalition is feasible so that classical cooperative games may be seen as special cooperative games with precedence constraints.

\footnotetext{
${ }^{*}$ The second author was supported by the Spanish Ministerio de Ciencia e Innovación under project ECO2009-11213, co-funded by the ERDF.

${ }^{\dagger}$ Paris School of Economics, University of Paris I, 106-112, Boulevard de l'Hôpital, 75103 Paris, France. E-mail: michel.grabisch@univ-paris1.fr

${ }^{\ddagger}$ Department of Business and Economics and COHERE, University of Southern Denmark, Campusvej 55, 5230 Odense M, Denmark. E-mail: psu@sam.sdu.dk
} 
The well-known fact that the core of a TU game with precedence constraints may be unbounded seems counterintuitive and has created several attempts to define a meaningful subset of the core that is bounded (see, e.g., Grabisch (2011)). The core of such a game is a convex polyhedral set that contains no lines, but, in contrast to the core of a classical TU game, it may have unbounded faces. Thus, attempts have been made to extract certain bounded faces of the core if the core itself is unbounded. In this paper, rather than trying to select one or some of the bounded faces, we consider the union of all bounded faces and call this union the "bounded core". An element $x$ of the core belongs to the bounded core if, for any player, each of her subordinates is a member of some coalition effective for $x$ that does not contain the player. Thus, in this sense each player takes the maximum of her subordinates.

The bounded core, though not convex, has many properties in common with the core of classical games. E.g., it is a connected, bounded, and closed set, and as a correspondence it is continuous. Faigle's (1989) generalization of the Bondareva-Shapley theorem may be used to show that the (bounded) core is nonempty whenever the underlying partial order generates a connected hierarchy. The bounded core may also be supported by its axiomatization by simple and intuitive axioms. Indeed, according to Hwang and Sudhölter (2001) the core is axiomatized by boundedness (BOUND), nonemptiness for zero-inessential two-person games (ZIG), anonymity, covariance under strategic equivalence (COV), the reduced game property (RGP), the converse reduced game property (CRGP), and the reconfirmation property. Suitable extensions and versions of the foregoing axioms characterize the bounded core if precedence constraints are possible. Moreover, the existing robustness results may be extended and even the bounded core of NTU games with precedence constraints may be characterized. If one restricts the attention to cooperative TU games on connected hierarchies then the core is axiomatized by BOUND, ZIG, COV, and some variants of RGP and CRGP. The unbounded core, though certainly less interesting, may be supported as the maximum solution that satisfies a suitably weakened version of BOUND (requiring that the payoffs to any feasible singleton are bounded from below) and (a subset of the) remaining axioms.

The paper is organized as follows. In Section 2 we recall basic definitions of a partially ordered set, of TU games with precedence constraints, and of the core. Moreover, the bounded core is formally introduced and an example is given that shows that the bounded core may be non-convex. In Section 3 we show that the bounded core of game with a connected hierarchy is nonempty and that the bounded core on the set of balanced games with coinciding precedence constraints is upper and lower hemicontinuous, whereas the core correspondence is lower hemicontinuous, but may fail to be upper hemicontinuous. In Section 4 we present the aforementioned generalizations of the robust axiomatizations of the core for classical TU and NTU games. Finally, in Section 5 we explicitly present the axiomatization of the bounded core when hierarchies are supposed to be connected. This axiomatization is much simpler and, hence, more appealing than in the general case. Also, we present examples that show that each of the employed axioms is logically independent of the remaining axioms. 


\section{Notation, Definitions, and Preliminaries}

A partially ordered set (poset) is a pair $(P, \preceq)$ such that $P$ is a nonempty finite set and $\preceq$ is a partial order on $P$, i.e., a reflexive, antisymmetric, and transitive binary relation on $P$. As usual, we write $x \preceq y$ for $(x, y) \in \preceq$ and use $x \prec y$ if $x \preceq y$ and $x \neq y$. If $x \prec y$ and there is no $z \in N$ such that $x \prec z \prec y$ then $y$ covers $x$, denoted by $x \prec \cdot y$. A chain in $(P, \preceq)$ is a sequence $\left(x_{0}, \ldots, x_{q}\right)$ such that $x_{0} \prec \cdots \prec x_{q}$ where $q$ is called the length of the chain. The height of a poset is the length of its longest chain.

Let $U$ be a set, the universe of players, containing, without loss of generality, $1, \ldots, k$ whenever $|U| \geqslant k$. A coalition is a finite nonempty subset of $U$. Let $N$ be a coalition and $(N, \preceq)$ be a poset. Then $S \subseteq N$ is a downset of $(N, \preceq)$ if $i \in S$ and $j \preceq i$ implies $j \in S$. Denote by $\mathcal{F} \preceq$ the set of downsets of $(N, \preceq)$. Note that $(\mathcal{F} \preceq, \subseteq)$ is a distributive lattice of height ${ }^{1}|N|$. By Birkhoff's representation theorem the opposite statement is also true: If $\mathcal{F} \subseteq 2^{N}$ and $(\mathcal{F}, \subseteq)$ is a distributive lattice of height $|N|$, then there exists a poset $(N, \preceq)$ such that $\mathcal{F}=\mathcal{F}$.

A (cooperative TU) game with precedence constraints (see Faigle and Kern (1992)) is a triple $(N, \preceq, v$ ) such that $N$ is a coalition, $(N, \preceq)$ is a poset, and $v: \mathcal{F} \preceq \rightarrow \mathbb{R}, v(\emptyset)=0$. Note that a classical TU game is a pair $(N, v)$ such that $v: 2^{N} \rightarrow \mathbb{R}, v(\emptyset)=0$. Hence, we may identify a game $(N, v)$ with $(N, \preceq, v)$ where $(N, \preceq)$ is the poset of height 0 .

Let $\Gamma$ denote the set of games with precedence constraints and $(N, \preceq, v) \in \Gamma$. Let

$$
X^{*}(N, \preceq, v)=\left\{x \in \mathbb{R}^{N} \mid x(N) \leqslant v(N)\right\} \text { and } X(N, \preceq, v)=\left\{x \in \mathbb{R}^{N} \mid x(N)=v(N)\right\}
$$

denote the set of feasible and Pareto efficient feasible payoffs (preimputations), respectively. We use $x(S)=\sum_{i \in S} x_{i}(x(\emptyset)=0)$ for every $S \in 2^{N}$ and every $x \in \mathbb{R}^{N}$ as a convention. Additionally, $x_{S}$ denotes the restriction of $x$ to $S$, i.e. $x_{S}=\left(x_{i}\right)_{i \in S}$, and we write $x=\left(x_{S}, x_{N \backslash S}\right)$.

The core of $(N, \preceq, v)$, denoted by $C(N, \preceq, v)$, is defined by

$$
C(N, \preceq, v)=\left\{x \in \mathbb{R}^{N} \mid x(N)=v(N) \text { and } x(S) \geqslant v(S) \text { for all } S \in \mathcal{F}^{\preceq}\right\}
$$

By its definition, the core of $(N, \preceq, v)$ is a convex polyhedral set. It is well known (see Derks and Gilles (1995)) that it does not contain lines. More precisely,

$$
C(N, \preceq, v)=\operatorname{conv}(\operatorname{ext}(C(N, \preceq, v))+C(N, \preceq, 0)
$$

where "conv" means "convex hull", "ext" means "set of extreme points", and "+" denotes "Minkowski sum". For any $S \subseteq N$, let ${ }^{N} \chi^{S}=\chi^{S} \in \mathbb{R}^{N}$ be the indicator function of $S$, i,e. $\chi_{i}^{S}=1$ for $i \in S$ and $\chi_{j}^{S}=0$ for $j \in N \backslash S$. If $(N, \preceq, v)$ is a classical game, i.e., if the height of $(N, \preceq)$ is 0 , then $C(N, \preceq, 0)=\{0\}$. Otherwise, i.e., if there exists a pair $(i, j) \in N$ such that $i \prec j$, then (see Derks and Gilles (1995))

$$
C(N, \preceq, 0)=\operatorname{cone}\left(\left\{\chi^{\{i\}}-\chi^{\{j\}} \mid i, j \in N, i \prec j\right\}\right),
$$

\footnotetext{
${ }^{1} \mathrm{~A}$ poset $(P, \preceq)$ is a lattice if for any $x, y \in P$ their supremum, denoted $x \wedge y$, and infimum, denoted $x \vee y$, exist. A lattice is distributive if $\wedge$ and $\vee$ satisfy distributivity.
} 
where "cone" denotes "convex cone generated by". For $i \prec j$, say $i=i_{0} \prec \cdots \prec i_{k}=j$,

$$
\chi^{\{i\}}-\chi^{\{j\}}=\sum_{\ell=0}^{k-1} \chi^{\left\{i_{\ell}\right\}}-\chi^{\left\{i_{\ell+1}\right\}}
$$

so that

$$
C(N, \preceq, 0)=\operatorname{cone}\left(\left\{\chi^{\{i\}}-\chi^{\{j\}} \mid i, j \in N, i \prec \cdot j\right\}\right)
$$

(also shown by Tomizawa (1983), see also Fujishige (2005, Th. 3.26)). We are now ready to define the bounded core.

Definition 2.1 Let $(N, \preceq, v) \in \Gamma$. The bounded core of $(N, \preceq, v)$, denoted by $C^{b}(N, \preceq, v)$, is the set of all elements $x \in C(N, \preceq, v)$ that satisfy the following condition for any $i, j \in N$ with $i \prec \cdot j$ : There is no $\varepsilon>0$ such that $x+\varepsilon\left(\chi^{\{j\}}-\chi^{\{i\}}\right) \in C(N, \preceq, v)$.

Thus, an element of the core is in the bounded core, if no player $j$ has an objection against any of the players $i$ she covers in the sense that as soon as some money is transferred from $i$ to $j$ this would result in a preimputation that does not belong to the core. Interpreting the partial order $\preceq$ as a hierarchy, we may say that $i$ is an immediate (or direct) subordinate of $j$ if $i \prec \cdot j$. Then, the bounded core is the set of core elements such that every player takes the maximum of her direct subordinates, in the sense that any money transfer from a subordinate to her boss would result in a payoff vector outside the core.

The following result is an immediate consequence of (2.2) and (2.4).

Corollary 2.2 If $(N, \preceq, v) \in \Gamma$, then

$$
C^{b}(N, \preceq, v)=\{x \in C(N, \preceq, v) \mid(\{x\}-C(N, \preceq, 0)) \cap C(N, \preceq, v)=\{x\}\} .
$$

Therefore, if $(N, \preceq, v)$ is a classical game, the bounded core coincides with the classical core.

Remark 2.3 Let $(N, \preceq, v) \in \Gamma$. According to Rockafellar (1970, Section 18) a closed convex set is the disjoint union of the relative interiors of its faces. Hence, any element of $C^{b}(N, \preceq, v)$ is in the interior of some face of $C(N, \preceq, v)$. We conclude that $C^{b}(N, \preceq, v)$ is the disjoint union of the relative interiors of the bounded faces of $C(N, \preceq, v)$, i.e., $C^{b}(N, \preceq, v)$ is the union of all bounded faces of $C(N, \preceq, v)$. Thus, the bounded core is connected.

The following example shows that the bounded core may be non-convex and, hence, a proper subset of the convex hull of the extreme points of the core (called "convex part of the core").

Example 2.4 Let $N=\{1, \ldots, 4\}$ and $\preceq$ be defined by $i \prec \cdot j$ iff $i=1$ and $j \in\{2,3\}$. Hence,

$$
F=F^{\preceq}=\{\emptyset,\{1\},\{4\},\{1,2\},\{1,3\},\{1,4\},\{1,2,3\},\{1,2,4\},\{1,3,4\}, N\} .
$$

Let $(N, \preceq, v)$ be defined by $v(\{1,2\})=v(\{1,3\})=2, v(N)=8$, and $v(S)=0$ for all other $S \in \mathcal{F}$. With $x=(0,4,4,0)$ and $y=(2,0,0,6)$, both $x$ and $y$ are elements of $C^{b}(N, \preceq v)$, but $\frac{x+y}{2}=(1,2,2,3) \in$ $C(N, v, \preceq) \backslash C^{b}(N, v, \preceq)$. 


\section{Properties of the core}

Let $N \subseteq U$ be a finite nonempty set. We recall Lemma 6.7 of Maschler, Peleg, and Shapley (1972) saying that a balanced collection of subsets is separating. A collection $\mathcal{B} \subseteq 2^{N}$ is balanced (over $N$ ) if positive real numbers $\delta_{S}, S \in \mathcal{B}$, exist such that $\sum_{S \in \mathcal{B}} \delta_{S} \chi^{S}=\chi^{N}$. The collection $\left(\delta_{S}\right)_{S \in \mathcal{B}}$ is a system of balancing weights. Note that for any balanced collection $\mathcal{B}$ with system $\left(\delta_{S}\right)_{S \in \mathcal{B}}$ of balancing weights, for $k, \ell \in N$,

$$
\begin{aligned}
& 1=\sum\left\{\delta_{S} \mid S \in \mathcal{B}, k \in S\right\}=\sum\left\{\delta_{S} \mid S \in \mathcal{B}, \ell \notin S \ni k\right\}+\sum\left\{\delta_{S} \mid S \in \mathcal{B}, k, \ell \in S\right\} \text { and } \\
& 1=\sum\left\{\delta_{T} \mid T \in \mathcal{B}, \ell \in T\right\}=\sum\left\{\delta_{T} \mid T \in \mathcal{B}, k \notin T \ni \ell\right\}+\sum\left\{\delta_{T} \mid T \in \mathcal{B}, k, \ell \in T\right\} .
\end{aligned}
$$

Thus, any balanced collection $\mathcal{B}$ is separating in the sense that the following condition is satisfied for all $k, \ell \in N$ : If there exists $S \in \mathcal{B}$ with $\ell \notin S \ni k$, then there exists $T \in \mathcal{B}$ with $k \notin T \ni \ell$.

A balanced collection $\mathcal{B}$ is minimal balanced if it does not contain a proper balanced subcollection. Note that a balanced collection is minimal balanced if and only if it has a unique system of balancing weights. Now, we are ready to formulate the well-known generalization of the sharp form of the Bondareva-Shapley theorem.

Theorem 3.1 (Faigle (1989)) Let $(N, \preceq, v) \in \Gamma$. A necessary and sufficient condition that the core of $(N, \preceq, v)$ is not empty is that for each minimal balanced collection $\mathcal{B} \in \mathcal{F}$ with $\mathcal{B} \neq\{N\}$,

$$
v(N) \geqslant \sum_{S \in \mathcal{B}} \delta_{S} v(S)
$$

where $\left(\delta_{S}\right)_{S \in \mathcal{B}}$ is the system of balancing weights for $\mathcal{B}$.

Let $(N, \preceq)$ be a poset and $i, j \in S \subseteq N$. We say that $i$ and $j$ are connected in $(S, \preceq)$ if there is a path in $S$ that connects $i$ and $j$, that is, if there exist $k \in \mathbb{N}$ and $i_{1}, \ldots, i_{k} \in N$ such that $i=i_{1}, j=i_{k}$, and, for each $\ell=1, \ldots, k-1$, either $i_{\ell} \prec i_{\ell+1}$ or $i_{\ell+1} \prec i_{\ell}$. Any $\emptyset \neq S \subseteq N$ may be partitioned into its connected components, and $S \subseteq N$ is connected if $S=\emptyset$ or $S$ consists of a single component.

Lemma 3.2 Let $(N, \preceq)$ be a poset and $N \subseteq U$.

(1) If $(N, \preceq)$ is connected (i.e., $N$ consists of a unique connected component), then $C(N, \preceq, v) \neq \emptyset$ for any $v: \mathcal{F} \preceq \rightarrow \mathbb{R}, v(\emptyset)=0$.

(2) If $(N, \preceq)$ is not connected, then there exists $v: \mathcal{F}^{\preceq} \rightarrow \mathbb{R}$ with $v(\emptyset)=0$ such that $C(N, \preceq, v)=\emptyset$.

\section{Proof:}

(1) Let $\mathcal{B} \subseteq \mathcal{F} \preceq$ be a balanced collection and $\left(\delta_{S}\right)_{S \in \mathcal{B}}$ be a system of balancing weights. In view of Theorem 3.1 it suffices to show that $N$ is the unique nonempty element of $\mathcal{B}$. Let $R \in \mathcal{B}, R \neq \emptyset$. 
Then there exists $i \in R$. In order to show that $R=N$, let $j \neq i$. As $(N, \preceq)$ is connected, there exist $k \in \mathbb{N}$ and $i_{0}, \ldots, i_{k} \in N$ such that $i_{0}=i, i_{k}=j$, and $i_{\ell} \prec i_{\ell+1}$ or $i_{\ell+1} \prec i_{\ell}$ for all $\ell=0, \ldots, k-1$. We show that $i_{\ell} \in R$ by induction on $\ell$. For $\ell=0$ nothing has to be proved. Assume that $i_{\ell} \in R$ for some $\ell<k$. If $i_{\ell+1} \prec i_{\ell}$, then $i_{\ell+1} \in R$ because $R \in \mathcal{F} \preceq$ is a downset. If $i_{\ell} \prec i_{\ell+1}$, then there exists $Q \in \mathcal{B}$ with $i_{\ell+1} \in Q$. As $\mathcal{F} \preceq$ is the set of downsets, any $S \in \mathcal{B}$ with $i_{\ell+1} \in S$ also contains $i_{\ell}$. As $\mathcal{B}$ is separating, $i_{\ell+1} \in R$.

(2) Let $(N, \preceq)$ be non connected and $v$ be a mapping on $\mathcal{F} \preceq$ with $v(\emptyset)=0$ that satisfies

$$
v(N)<\sum\{v(S) \mid S \text { is a connected component of }(N, \preceq)\}
$$

Clearly, $C(N, \preceq, v)=\emptyset$.

q.e.d.

For the rest of this section we fix a poset $(N, \preceq), N \subseteq U$, and identify a TU game $(N, \preceq, v)$ simply by its coalition function $v: \mathcal{F} \rightarrow \mathbb{R}$, where $\mathcal{F}=\mathcal{F} \preceq$. Denote by $\Gamma^{\preceq}$ the set of these games and let $\Gamma_{b}^{\preceq}=\left\{v \in \Gamma^{\preceq} \mid C(v) \neq \emptyset\right\}$ (those games that are balanced). Moreover, let $\Gamma^{N}$ and $\Gamma_{b}^{N}$ be the set of classical and of classical balanced TU games with player sets $N$, respectively. We recall that for any $v \in \Gamma^{N}, C(v)=C^{b}(v)$.

Let $v \in \Gamma^{\prec}$. For any $i \in N$, let

$$
b_{i}(N, \preceq, v)=b_{i}(v)=\min \left\{v(S \cup\{i\})-v(S) \mid S, S \cup\{i\} \in \mathcal{F}^{\preceq}, i \notin S\right\} .
$$

That is, $b_{i}(v)$ is $i$ 's minimal marginal contribution.

Lemma 3.3 Let $v \in \Gamma \preceq$ and $w \in \Gamma^{N}$ such that $w(S)=v(S)$ for all $S \in \mathcal{F}$. Then $(i) C(w) \subseteq C(v)$ and (ii) if $w(T) \leqslant \sum_{i \in T} b_{i}(v)$ for all $T \in 2^{N} \backslash \mathcal{F}$, then $C^{b}(v) \subseteq C(w)$.

Proof: The first statement is obviously true. In order to show the second inclusion, let $x \in C^{b}(v)$ and $i \in N$. If $i$ is a minimal element, then $x_{i} \geqslant v(\{i\}) \geqslant b_{i}(v)$. Otherwise there exists $j \in N$ such that $j \prec \cdot i$. As $x+\varepsilon\left(\chi^{\{i\}}-\chi^{\{j\}}\right) \notin C(v)$ for any $\varepsilon>0$, there exists $S \in \mathcal{F}$ such that $i \notin S \ni j$ and $v(S)=x(S)$. Hence, $0 \geqslant v(S \cup\{i\})-x(S \cup\{i\})=v(S \cup\{i\})-v(S)-x_{i}$ so that $x_{i} \geqslant v(S \cup\{i\})-v(S) \geqslant b_{i}(v)$. We conclude $x(S) \geqslant \sum_{i \in S} b_{i}(v)$ for any $S \in 2^{N}$.

q.e.d.

We recall that a collection $\mathcal{N} \subseteq \mathcal{F}$ is normal (with respect to (w.r.t.) $(N, \preceq)$ ) if

$$
C_{\mathcal{N}}(v)=\left\{x \in \mathbb{R}^{N} \mid x(N)=v(N), \quad x(S) \geqslant v(S) \quad \forall S \in \mathcal{F}, \quad x(S)=v(S) \quad \forall S \in \mathcal{N}\right\}
$$

is bounded (see Grabisch (2011)).

Proposition 3.4 Let $\mathcal{F}=\mathcal{F} \preceq$ for some fixed $(N, \preceq)$. Then for any game $v \in \Gamma_{\bar{b}}^{\preceq}$

$$
C^{b}(v)=\bigcup\left\{C_{\mathcal{N}}(v) \mid \mathcal{N} \subseteq \mathcal{F} \text { is a normal collection }\right\}
$$


Proof: Take $x \in C^{b}(v)$. As in the proof of Lemma 3.3, for any pair $(i, j)$ with $j \prec \cdot i$, there exists $S \in \mathcal{F}$, $i \notin S \ni j$, such that $x(S)=v(S)$. By Lemma 2 of Grabisch (2011), the collection of these $S$ form a normal collection $\mathcal{N}$. Hence $x \in C_{\mathcal{N}}(v)$.

Conversely, take a normal collection $\mathcal{N}$ and $x \in C_{\mathcal{N}}(v)$. Then by Lemma 2 again, for any pair $(i, j)$ with $j \prec \cdot i$, there exists $S \in \mathcal{N}$, such that $i \notin S \ni j$, and $x(S)=v(S)$. Hence for any $\epsilon>0$, taking $x^{\prime}=x+\epsilon\left(\chi^{i}-\chi^{j}\right)$, we would have $x^{\prime}(S)<v(S)$, proving that $x \in C^{b}(v)$.

q.e.d.

Note that if $(N, \preceq)$ has height 0 (i.e., $(N, \preceq)$ is a classical game), then the empty collection $(\mathcal{N}=\emptyset)$ is normal, so that Proposition 3.4 recovers the fact that $C^{b}(v)=C(v)$ for classical games.

Now, we consider the set-valued functions $C^{b}: \Gamma_{\bar{b}}^{\prec} \rightrightarrows \mathbb{R}^{N}$ and $C: \Gamma_{\bar{b}}^{\prec} \rightrightarrows \mathbb{R}^{N}$. It is well-known (see, e.g., Peleg and Sudhölter (2007)) that $C: \Gamma_{b}^{N} \rightrightarrows \mathbb{R}^{N}$ is continuous, i.e., upper hemicontinuous (uhc) and lower hemicontinuous (lhc).

Let $\mathcal{S} \preceq=\{(i, j) \in N \times N \mid i \prec \cdot j\}$. For $v \in \Gamma^{\prec}$ and $x \in C(v)$ define

$$
d(x, v)=\max _{(i, j) \in \mathcal{S} \preceq} \min \{x(S)-v(S) \mid S \in \mathcal{F}, j \notin S \ni i\}
$$

and

$$
\beta(x, v)=\left|\left\{(i, j) \in \mathcal{S}^{\preceq} \mid \min \{x(S)-v(S) \mid S \in \mathcal{F}, j \notin S \ni i\}>0\right\}\right| .
$$

Hence, if $x \in C(v)$, then (i) $d(x, v), \beta(x, v) \geqslant 0$ and (ii) $x \in C^{b}(v)$ iff $d(x, v)=0$ iff $\beta(x, v)=0$.

Lemma 3.5 Let $v \in \Gamma^{\preceq}$. If $x \in C(v)$ such that $\beta(x, v)>0$, then there exists $y \in C(v)$ such that $d(y, v) \leqslant d(x, v), \beta(y, v)<\beta(x, v)$, and $\|y-x\|_{\infty} \leqslant d(x, v)$.

Proof: Choose any $(i, j) \in \mathcal{S} \preceq$ such that $\varepsilon:=\min \{x(S)-v(S) \mid S \in \mathcal{F}, j \notin S \ni i\}>0$. Define $y=x+\varepsilon\left(\chi^{\{j\}}-\chi^{\{i\}}\right)$. Then $\varepsilon \leqslant d(x, v), \beta(y, v) \leqslant \beta(x, v)-1$, and $\|y-x\|_{\infty}=\varepsilon$.

q.e.d.

Theorem 3.6 The set-valued function $C^{b}: \Gamma_{b}^{\prec} \rightrightarrows \mathbb{R}^{N}$ is continuous.

Proof: uhc: We first show that the graph of $C^{b}, \operatorname{Gr}\left(C^{b}\right)=\left\{(v, x) \in \Gamma_{b}^{\prec} \times \mathbb{R}^{N} \mid x \in C^{b}(v)\right\}$ is closed. For this purpose, let $v^{t} \in \Gamma_{b}^{\prec}$ and $x^{t} \in C^{b}\left(v^{t}\right)$ for $t \in \mathbb{N}$ so that $\lim _{t \rightarrow \infty} v^{t}=v$ and $\lim _{t \rightarrow \infty} x^{t}=x$. Clearly, $x \in C(v)$. For any $(i, j) \in \mathcal{S}^{\preceq}$ let $S_{(i, j)}^{t} \in \mathcal{F}$ such that $j \notin S \ni i$ and $x^{t}(S)=v^{t}(S)$. As $|\mathcal{F}|$ is finite, for any $(i, j) \in \mathcal{S} \preceq$ there exists a collection $S_{(i, j)} \in \mathcal{F}$ such that $S_{(i, j)}^{t}=S_{(i, j)}$ for infinitely many $t \in \mathbb{N}$. Hence, $x\left(S_{(i, j)}\right)=v\left(S_{(i, j)}\right)$ for all $(i, j) \in \mathcal{S}^{\preceq}$ and $x \in C^{b}(v)$.

It suffices to show that $C^{b}$ is a bounded set valued function, i.e., the image of a compact subset $\Gamma^{\prime}$ of $\Gamma_{b}^{\prec}$ is bounded. Now, as $\Gamma^{\prime}$ is compact, there exists $t \leqslant 0$ such that $t \leqslant b_{i}(v)$ for all $i \in N$ and $v \in \Gamma^{\prime}$. Let $\Gamma^{\prime \prime}$ be the set of all classical games $w$ such that the restriction of $w$ to $\mathcal{F}$ belongs to $\Gamma^{\prime}$ and $w(S)=|N| t$ for all $S \in 2^{N} \backslash \mathcal{F}$. Then $\Gamma^{\prime \prime}$ inherits compactness from $\Gamma^{\prime}$. It is well-known (see Peleg and Sudhölter (2007, Chapter 9)) that the core correspondence on classical balanced games is bounded. By (ii) of Lemma 3.3, $C^{b}$ is bounded. 
Moreover, a closed and bounded set-valued function is uhc.

lhc: Let $v, v^{t} \in \Gamma_{\bar{b}}^{\prec}$ for $t \in \mathbb{N}$ such that $\lim _{t \rightarrow \infty} v^{t}=v$, and let $x \in C^{b}(v)$. It suffices to construct a sequence $x^{t} \in C^{b}\left(v^{t}\right)$ such that $\lim _{t \rightarrow \infty} x^{t}=x$. Define classical games $w^{t}$ such that $w^{t}(S)=v^{t}(S)$ for all $S \in \mathcal{F}$ and $w^{t}(T)=\sum_{i \in T} b_{i}\left(v^{t}\right)$ for all $T \in 2^{N} \backslash T$. Moreover, let $w \in \Gamma^{N}$ be defined by $w(S)=v(S)$ for all $S \in \mathcal{F}$ and $w(T)=\sum_{i \in T} b_{i}(v)$ for all $T \in 2^{N} \backslash T$. Then $\lim _{t \rightarrow \infty} w^{t}=w$ and, by Lemma $3.3, x \in C(w)$. As $C$ is lhc on classical games, there exist $y^{t} \in C\left(w^{t}\right)$ such that $\lim _{t \rightarrow \infty} y^{t}=x$. By Lemma 3.3, $y^{t} \in C\left(v^{t}\right)$. By Lemma 3.5 and the triangle inequality there exist $x^{t} \in C^{b}\left(v^{t}\right)$ such that $\left\|x^{t}-y^{t}\right\|_{\infty} \leqslant\left|\mathcal{S}^{\prec}\right| d\left(y^{t}, v\right)$. As $\lim _{t \rightarrow \infty} v^{t}=v$ and $\lim _{t \rightarrow \infty} y^{t}=x, \lim _{t \rightarrow \infty} d\left(y^{t}, v\right)=d(x, v)=0$. Thus, $\lim _{t \rightarrow \infty} x^{t}=x$. q.e.d. The set-valued function $C: \Gamma_{b}^{\prec} \rightrightarrows \mathbb{R}^{N}$ inherits lhc from $C^{b}$. Indeed, if $x \in C(v)$, then there exist $y \in C^{b}(v)$ and $z \in C(0)$ such that $x=y+z$. Now, if $\lim _{t \rightarrow \infty} v^{t}=v$, then by lhc of $C^{b}$ there exist $y^{t} \in C^{b}\left(v^{t}\right), t \in \mathbb{N}$, such that $\lim _{t \rightarrow \infty} y^{t}=y$. Moreover, $x^{t}:=y^{t}+z \in C\left(v^{t}\right)$ and $\lim _{t \rightarrow \infty} x^{t}=x$.

However, $C$ is not bounded unless the height of $(N, \preceq)$ is 0 . We now present an example that shows that $C$ may not be continuous (uhc) even in the case $|N|=2$.

Example 3.7 Let $N=\{1,2\}$ and $1 \prec 2$. Let $v$ and $v^{t}$ be defined by $v^{t}(\{1\})=v(\{1\})=v(N)=0$ and $v^{t}(N)=\frac{1}{t}$ for all $t \in \mathbb{N}$. Then $\lim _{t \rightarrow \infty} v^{t}=v, C(v)=\left\{x \in \mathbb{R}^{2} \mid x_{1} \geqslant 0, x_{2}=-x_{1}\right\}$, and $C\left(v^{t}\right)=\left\{x \in \mathbb{R}^{2} \mid x_{1} \geqslant 0, x_{2}=-x_{1}+\frac{1}{t}\right\}$. Let $U=\left\{x \in \mathbb{R}^{2} \mid x_{2}<-x_{1}+e^{-x_{1}}\right\}$. Then $U$ is an open set that contains $C(v)$. However, for any $t \in \mathbb{N}$ there exist $x_{1}>0$ such that $\frac{1}{t}>e^{-x_{1}}$ so that $\left(x_{1},-x_{1}+\frac{1}{t}\right) \in C\left(v^{t}\right) \backslash U$. Therefore, $C$ is not uhc.

\section{Axiomatization of the bounded core}

A solution on $\Gamma^{\prime} \subseteq \Gamma$ is a mapping $\sigma$ that associates with each $(N, \preceq, v) \in \Gamma^{\prime}$ a set $\sigma(N, \preceq, v) \subseteq$ $X^{*}(N, \preceq, v)$. Let $\sigma$ be a solution on some $\Gamma^{\prime} \subseteq \Gamma$. Then the restriction of $\sigma$ to any $\Gamma^{\prime \prime}$ is a solution on $\Gamma^{\prime \prime}$ so that we say that $\sigma$ is a solution on $\Gamma^{\prime \prime}$, too. If $\Gamma^{\prime}$ is not specified, then we mean that $\sigma$ is a solution on $\Gamma$ (and any of its subsets).

We now generalize some well-known properties of a solution on a set of classical games.

A solution $\sigma$ on $\Gamma^{\prime} \subseteq \Gamma$ satisfies:

(1) Pareto optimality (PO) if $\sigma(N, \preceq, v) \subseteq X(N, \preceq, v)$ for all $(N, \preceq, v) \in \Gamma^{\prime}$.

(2) Covariance under strategic equivalence (COV) if, for all $(N, \preceq, v),(N, \preceq, w) \in \Gamma^{\prime}, \alpha>0$, and $\beta \in$ $\mathbb{R}^{N}$, the following condition is valid: If $w(S)=\alpha v(S)+\beta(S)$ for all $S \in \mathcal{F} \preceq$, then $\sigma(N, \preceq, w)=$ $\alpha \sigma(N, \preceq, v)+\beta$.

(3) Anonymity (AN) if, for all $(N, \preceq, v) \in \Gamma^{\prime}$ and all injective mappings $\pi: N \rightarrow U$ the following condition is valid: If $\left(\pi(N), \preceq^{\prime}, \pi v\right) \in \Gamma^{\prime}$, where $\pi(i) \preceq^{\prime} \pi(j)$ iff $i \preceq j,(\pi v)(\pi(S))=v(S)$ for all 
$S \subseteq \mathcal{F}^{\preceq}$, and $\pi(x)=y \in \mathbb{R}^{\pi(N)}$ is defined by $y_{\pi(i)}=x_{i} \forall x \in \mathbb{R}^{N}, \forall i \in N$, then $\sigma\left(\pi(N), \preceq^{\prime}, \pi v\right)=$ $\pi(\sigma(N, \preceq, v))$.

(4) Boundedness (BOUND) if $\sigma(N, \preceq, v)$ is a bounded set for all $(N, \preceq, v) \in \Gamma^{\prime}$.

(5) The two-person zero-inessential game property (ZIG) if $\sigma(N, \preceq, 0) \neq \emptyset$ for all $(N, \preceq, 0) \in \Gamma^{\prime}$ satisfying $|N|=2$.

In order to generalize various reduced game properties, we first have to define the generalization of the Davis-Maschler reduced game of a classical game. Let $(N, \preceq, v) \in G$ and $\emptyset \neq S \subseteq N$. Let $\left(S, \preceq_{S}\right)$ denote the sub-poset of $(N, \preceq)$ on $S$, i.e., the intersection of $\preceq$ and $S \times S$. Note that $\mathcal{F} \preceq S=\{T \cap S \mid T \in \mathcal{F} \preceq\}$.

Remark 4.1 Let $(N, \preceq, v) \in \Gamma$ and $\emptyset \neq S \in \mathcal{F}^{\preceq}$. Then $\mathcal{F}^{\preceq}=\left\{T \in \mathcal{F}^{\preceq} \mid T \subseteq S\right\}$. Hence, with $v_{S}(T)=v(T)$ for all $T \in \mathcal{F} \preceq, T \subseteq S$, the game $\left(S, \preceq_{S}, v_{S}\right)$ is the subgame of $(N, \preceq, v)$ w.r.t. S. Slightly abusing notation we use $v_{S}=v$ and $\preceq_{S}=\preceq$ in this case.

Let $x \in \mathbb{R}^{N}$. The reduced game of $(N, \preceq, v)$ w.r.t. $S$ and $x$ is the game $\left(S, \preceq{ }_{S}, v{ }_{S, x}\right)$ defined by

$$
v \preceq(T)= \begin{cases}0 & , \text { if } T=\emptyset, \\ v(N)-x(N \backslash S) & , \text { if } T=S, \\ \max \left\{v(R)-x(R \backslash T) \mid R \in \mathcal{F}^{\preceq}, R \cap S=T\right\} & , \text { if } T \in \mathcal{F}^{\preceq} \backslash\{\emptyset, S\} .\end{cases}
$$

The solution $\sigma$ satisfies the

(6) reduced game property (RGP) if the following condition holds: If $(N, \preceq, v) \in \Gamma^{\prime}, \emptyset \neq S \subseteq N$, and $x \in \sigma(N, \preceq, v)$, then $\left(S, \preceq_{S}, v \preceq_{S, x}\right) \in \Gamma^{\prime}$ and $x_{S} \in \sigma\left(S, \preceq_{S}, v{ }_{\bar{S}, x}\right)$;

(7) converse reduced game property (CRGP) if the following condition holds: If $(N, \preceq, v) \in \Gamma^{\prime},|N| \geqslant$ $2, x \in X(N, \preceq, v),\left(S, \preceq_{S}, v \widehat{S}_{x}\right) \in \Gamma^{\prime}$ and $x_{S} \in \sigma\left(S, \preceq_{S}, v \preceq_{S}, x\right)$ for all $S \subseteq N$ with $|S|=2$, then $x \in \sigma(N, \preceq, v)$;

(8) reconfirmation property $(\mathrm{RCP})$ if the following condition holds for every $(N, \preceq, v) \in \Gamma^{\prime}$, every $x \in$

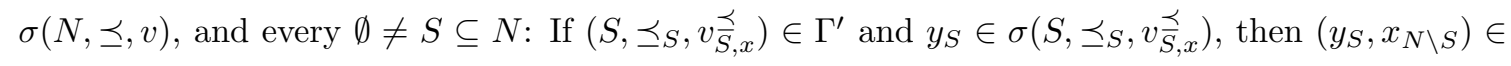
$\sigma(N, \preceq, v)$.

Remark 4.2 On any set of classical games the core satisfies all of the foregoing eight axioms except RGP, and it satisfies RGP if the class of classical games is closed under reduction w.r.t. core elements. Similar proofs show the same results for the core on a set of games with precedence constraints, with one exception, namely BOUND. However, BOUND is a crucial assumption in the axiomatization of the core by Hwang and Sudhölter (2001).

Lemma 4.3 The bounded core satisfies AN, COV, BOUND and CRGP on any $\Gamma^{\prime} \subseteq \Gamma$, and it satisfies RGP on any set $\Gamma^{\prime} \subseteq \Gamma$ that is closed under reduction w.r.t. elements of the bounded core. 
Proof: Clearly, $C^{b}$ inherits AN and COV from $C$. Let $(N, \preceq, v) \in \Gamma$. By Proposition 3.4, BOUND is proved. Let $x \in C(N, \preceq, v)$ and $\emptyset \neq S \subseteq N$. Define $u=v_{\widehat{S}, x}$. By Remark 4.2, $x_{S} \in C\left(S, \preceq_{S}, u\right)$. If $x_{S} \notin C^{b}\left(S, \preceq_{S}, u\right)$, then there exist $i, j \in S$ and $\varepsilon>0$ such that $i \prec_{S} \cdot j$ and $y_{S}:=x_{S}+\varepsilon\left({ }^{S} \chi^{\{j\}}-{ }^{S} \chi^{\{i\}}\right) \in$ $C\left(S, \preceq_{S}, u\right)$. By Remark 4.2, the core satisfies RCP so that $\left(y_{S}, x_{N \backslash S}\right)=x+\varepsilon\left({ }^{N} \chi\{j\}-{ }^{N} \chi\{i\}\right)$ belongs to $C(N, \preceq, v)$, so that $x \notin C^{b}(N, \preceq, v)$. Hence, the bounded core satisfies RGP provided that the reduced games w.r.t. bounded core elements belong to the set of games under consideration. In order to show CRGP, assume that $|N| \geqslant 2$ and that $x \in X(N, \preceq, v)$ and $x_{S} \in C\left(S, \preceq_{S}, v \breve{S}, x^{\prime}\right)$ for all $S \subseteq N$ with $|S|=2$. By Remark 4.2, $x \in C(N, \preceq, v)$. If $x \notin C^{b}(N, \preceq, v)$, then there exist $i, j \in N$ and $\varepsilon>0$ such that $i \prec j$ and $y:=x+\varepsilon\left(\chi^{\{j\}}-\chi^{\{i\}}\right) \in C(N, \preceq, v)$. With $S=\{i, j\}, y_{S} \in C\left(S, \preceq_{S}, v \frac{\prec}{\widehat{S}, y}\right)$ by Remark 4.2. As $v \stackrel{\swarrow}{\widehat{S}, y}=v_{\widehat{S}, x}^{\prec}$ and $y_{S}=x_{S}+\varepsilon\left({ }^{S} \chi^{\{j\}}-{ }^{S} \chi^{\{i\}}\right), x_{S} \notin C^{b}\left(S, \preceq_{S}, v_{\bar{S}, x}^{\prec}\right)$ so that CRGP follows.

q.e.d.

The following example shows that the bounded core may not satisfy RCP.

Example 2.4 cont. Let $S=\{1,4\}, \preceq^{\prime}=\preceq_{S}$, and $u=v_{\bar{S}, y}$. Then $u(\{1\})=2, u(\{4\})=0$, and $u(S)=8$ so that with $z_{1}=8$ and $z_{4}=0, z \in C(S, u)=C\left(S, \preceq^{\prime}, u\right)=C^{b}\left(S, \preceq^{\prime}, u\right)$. However, $\left(z, y_{N \backslash S}\right)=(8,0,0,0) \in$ $C(N, \preceq, v) \backslash C^{b}(N, \preceq, v)$.

Hence, we use a weaker property than RCP. Let $(N, \preceq)$ be a poset and $i, j \in N$. We say that a solution $\sigma$ on $\Gamma^{\prime} \subseteq \Gamma$ satisfies the reconfirmation property w.r.t. classical games $\mathrm{RCP}^{c g}$ if it satisfies (8) for all classical games $(N, \preceq, v) \in \Gamma^{\prime}$ (i.e., the height of $(N, \preceq)$ is 0 ). Hence, on sets of classical TU games, RCP and $\mathrm{RCP}^{c g}$ cannot be distinguished.

Note that the bounded core coincides with the core on any set of classical games so that it satisfies $\mathrm{RCP}^{c g}$ on any set of games with precedence constraints.

Till the end of this section we assume that $|U| \geqslant 5$.

Theorem 4.4 The bounded core is the unique solution on $\Gamma$ that satisfies $\mathrm{ZIG}, \mathrm{AN}, \mathrm{COV}, \mathrm{RGP}, \mathrm{RCP}^{c g}$, CRGP, and BOUND.

Proof: By definition of the bounded core, $0 \in C^{b}(N, \preceq, 0)$ for any flat game $(N, \preceq, 0) \in \Gamma$. Hence, $C^{b}$ satisfies ZIG. By Lemma 4.3, the bounded core satisfies the remaining axioms as well. In order to show the uniqueness part, let $\sigma$ be a solution that satisfies the seven foregoing axioms. Hwang and Sudhölter (2001, Theorem 4.1) show that $\sigma$ coincides with the core on the set of classical games provided $|U| \geqslant 5$. Hence, by CRGP and RGP, it suffices to show that $\sigma$ coincides with the bounded core for any two-person game that is not a classical game. Indeed, assume that this property holds. Take $x \in \sigma(N, \preceq, v)$. By RGP of $\sigma$, for any $S \subseteq N,|S|=2, x_{S} \in \sigma\left(S, \preceq_{S}, u\right)=C^{b}\left(S, \preceq_{S}, u\right)$, where $u$ is the reduced game. Then by CRGP of $C^{b}, x \in C^{b}(N, \preceq, v)$. The converse is obtained by permuting $\sigma$ and $C^{b}$. Let $(N, \preceq, v) \in \Gamma$ with $N=\{i, j\}$ and $i \prec j$. By COV we may assume that $v(\{i\})=v(N)=0$. By ZIG there exists $x \in \sigma(N, \preceq, v)$. By COV,$\alpha x \in \sigma(N, \preceq, \alpha v)$ for any $\alpha>0$. As $\alpha v=v=0, x=0$ by BOUND. Hence, $\sigma(N, \preceq, v)=C^{b}(N, \preceq, v)$. 
In order to characterize the core, we basically replace BOUND by individual rationality: A solution $\sigma$ in a set $\Gamma^{\prime} \subseteq \Gamma$ is

(9) individually rational (IR) if, for any $(N, \preceq, v) \in \Gamma^{\prime}, x \in \sigma(N, \preceq, v)$, and $i \in N$, the following property holds: If $\{i\} \in \mathcal{F} \preceq$, then $x_{i} \geqslant v(\{i\}$;

(4') bounded w.r.t. singletons $\left(\mathrm{BOUND}^{s}\right)$ if for any $(N, \preceq, v) \in \Gamma^{\prime}$, the restriction of $\sigma(N, \preceq, v)$ to the set $\left\{i \in N \mid\{i\} \in \mathcal{F}^{\preceq}\right\}$ is bounded from below ${ }^{2}$.

Clearly IR implies BOUND B $^{s}$

Moreover, for classical TU games, BOUND and BOUND ${ }^{s}$ are equivalent. Let $\sigma$ and $\sigma^{\prime}$ be solutions on $\Gamma^{\prime} \subseteq \Gamma$. We say that $\sigma^{\prime}$ is a subsolution of $\sigma$ if $\sigma^{\prime}(N, \preceq, v) \subseteq \sigma(N, \preceq, v)$ for all $(N, \preceq, v) \in \Gamma^{\prime}$.

Lemma 4.5 Any solution $\sigma$ that satisfies ZIG, AN, COV, RGP, RCP ${ }^{c g}$, CRGP, and BOUND ${ }^{s}$ is a subsolution of the core.

Proof: Again by Theorem 4.1 of Hwang and Sudhölter (2001), $\sigma$ coincides with the core for any classical game. By RGP and CRGP (proceeding similarly as in the corresponding part of the proof of Theorem 4.4), it suffices to show that $\sigma(N, \preceq, v) \subseteq C(N, \preceq, v)$ for any two-person game $(N, \preceq, v)$ that is not a classical game. Let $N=\{i, j\}, i \prec j$, and $x \in \sigma(N, \preceq, v)$. By COV, we may assume that $v(\{i\})=v(N)=0$,

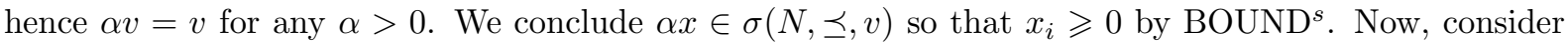
$S=\{i\}$, denoting the reduced game by $u$. By RGP, $x_{i} \in \sigma\left(\{i\}, \preceq_{\{i\}}, u\right)$. Since the reduced game is a classical game, $x_{i}=u(\{i\})=v(\{i, j\})-x_{j}=-x_{j}$. Therefore, $x \in C(N, \preceq, v)$.

q.e.d.

Corollary 4.6 The core is the maximum solution that satisfies ZIG, AN, COV, RGP, RCP ${ }^{c g}$, $\mathrm{CRGP}^{\text {, }}$ and $\mathrm{BOUND}^{s}$.

Several other characterizations of Hwang and Sudhölter (2001) may be generalized to games with precedence constraints as well. Indeed, $\Gamma_{b}$ denote the set of balanced games in $\Gamma$. Moreover, let $\Gamma_{t b}$ denote the set of totally balanced games in $\Gamma$. A game $(N, \preceq, v) \in \Gamma$ is totally balanced if, for any $\emptyset \neq S \in \mathcal{F} \preceq$, the subgame $(S, \preceq, v)$ (see Remark 4.1 ) is balanced.

Note that a reduced game of a game in $\Gamma_{t b}$ w.r.t. a core element may not be balanced. Therefore, we shall employ the weak reduced game property defined as follows. A solution on $\Gamma^{\prime} \subseteq \Gamma$ satisfies the

(6') weak reduced game property (WRGP) if the following condition holds: If $(N, \preceq, v) \in \Gamma^{\prime}, \emptyset \neq S \subseteq$ $N,|S| \leqslant 2$, and $x \in \sigma(N, \preceq, v)$, then $\left(S, \preceq_{S}, v \preceq_{S, x}\right) \in \Gamma^{\prime}$ and $x_{S} \in \sigma\left(S, \preceq_{S}, v{ }_{\bar{S}, x}\right)$.

Theorem 5.1 of Hwang and Sudhölter (2001) may be generalized as follows.

\footnotetext{
${ }^{2}$ I.e., there exists $\beta \in \mathbb{R}$ such that $x_{i} \geqslant \beta$ for any $i \in N$ such that $\{i\} \in \mathcal{F}$ and any $x \in \sigma(N, \preceq, v)$.
} 
Proposition 4.7 Let $\Gamma_{t b} \subseteq \Gamma^{\prime} \subseteq \Gamma$ such that $\Gamma^{\prime}$ does not contain non-balanced two-person games. Then the bounded core on $\Gamma^{\prime}$ is the unique solution that satisfies ZIG, COV, WRGP, RCP ${ }^{c g}$, CRGP, and BOUND.

It should be noted that the results on the core of NTU games (see Section 7 of the aforementioned paper) may be generalized to NTU games with precedence constraints in a canonical way. Moreover, examples are presented that show that each axiom employed in the various characterizations is logically independent of the remaining axioms. Suitable modifications of these examples may be used to show the logical independence of the axioms employed in Theorem 4.4 and Proposition 4.7. Finally it should be remarked that the assumption $|U| \geqslant 5$ is already crucial for the results on classical games.

\section{The bounded core for games with connected hierarchies}

We say that $(N, \preceq, v) \in \Gamma$ has a connected hierarchy if $(N, \preceq)$ is connected. Let $\Gamma^{c h}$ denote the set of all TU games $(N, \preceq, v)$ that have connected hierarchies. This section is devoted to an axiomatization of the bounded core for games with connected hierarchies.

In order to characterize the core on $\Gamma^{c h}$, the following variant of the reduced game property is useful. A solution $\sigma$ on $\Gamma^{\prime} \subseteq \Gamma$ satisfies

$\left(6^{c c}\right)$ reduced game property w.r.t. connected coalitions $\left(\mathrm{RGP}^{c c}\right)$ if the following condition holds: If $(N, \preceq, v) \in \Gamma^{\prime}, \emptyset \neq S$ connected w.r.t. $(N, \preceq)$, and $x \in \sigma(N, \preceq, v)$, then $\left(S, \preceq_{S}, v_{\bar{S}, x}\right) \in \Gamma^{\prime}$ and

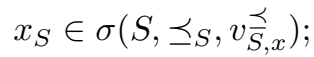

$\left(6^{\prime c c}\right)$ weak reduced game property w.r.t. connected coalitions (WRGP ${ }^{c c}$ ) if the following condition holds: If $(N, \preceq, v) \in \Gamma^{\prime}, \emptyset \neq S$ connected w.r.t. $(N, \preceq),|S| \leqslant 2$, and $x \in \sigma(N, \preceq, v)$, then $\left(S, \preceq_{S}, v_{\widehat{S}, x}\right) \in \Gamma^{\prime}$ and $x_{S} \in \sigma\left(S, \preceq_{S}, v_{\bar{S}, x}^{\swarrow}\right)$;

(7') converse reduced game property w.r.t. connected coalitions (CRGP ${ }^{c c}$ ) if the following condition

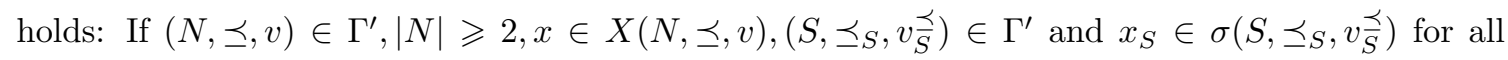
connected $S \subseteq N$ with $|S|=2$, then $x \in \sigma(N, \preceq, v)$;

(5’) nonemptiness (NEM) if $\sigma(N, \preceq, v) \neq \emptyset$ for all $(N, \preceq, v) \in \Gamma^{\prime}$.

Lemma 5.1 On $\Gamma^{c h}$ the bounded core satisfies $\mathrm{RGP}^{c c}, \mathrm{CRGP}^{c c}$, and NEM.

Proof: Let $(N, \preceq, v) \in \Gamma^{c h}$. In order to show $\operatorname{RGP}^{c c}$, let $\emptyset \neq S$ be a connected coalition, and $x \in$ $C^{b}(N, \preceq, v)$. Then $S$ remains connected w.r.t. $\left(S, \preceq_{S}\right)$ so that, as $C^{b}$ satisfies RGP, $x_{S} \in C^{b}\left(S, \preceq_{S}, v \frac{\swarrow}{S}, x\right)$. In order to show $\mathrm{CRGP}^{c c}$, assume that $|N| \geqslant 2$ and $x \in X(N, \preceq, v) \backslash C^{b}(N, \preceq, v)$. If $x \notin C(N, \preceq, v)$, there exists $T \in \mathcal{F} \preceq$ such that $v(T)>x(T)$. As $\emptyset \neq T \neq N$ and $N$ are connected, there exist $i \in T$ and $j \in N \backslash T$ such that $i \prec \cdot j$ so that $S=\{i, j\}$ is connected. Let $u=v_{\bar{S}, x}$. We have $\left(S, \preceq_{S}, u\right) \in \Gamma^{c h}$. 
Moreover, $x_{S} \notin C\left(S, \preceq_{S}, u\right)$, therefore $x_{S} \notin C^{b}\left(S, \preceq_{S}, u\right)$. Indeed, $u(\{i\}) \geqslant v(T)-x(T \backslash i)$. Since $v(T)>x(T)$, this entails $u(\{i\})>x_{i}$. If $x \in C(N, \preceq, v) \backslash C^{b}(N, \preceq, v)$, then, proceeding as in the proof of Lemma 4.3, there exist $k, \ell \in N$ such that $k \prec \cdot \ell$ and $\varepsilon>0$ such that $y:=x+\varepsilon\left(\chi^{\ell}-\chi^{k}\right) \in C(N, \preceq, v)$. As $S=\{k, \ell\}$ is connected, $\left(S, \preceq_{S}, v v_{\bar{S}, x}^{\preceq}\right) \in \Gamma^{c h}$. As $y_{S} \in C\left(S, \preceq_{S}, v_{\bar{S}, x}^{\prec}\right), x_{S} \notin C^{b}\left(S, \preceq_{S}, v_{\bar{S}, x}\right)$. We conclude that $C^{b}$ satisfies $\mathrm{CRGP}^{c c}$.

NEM follows from Lemma $3.2(1)$.

q.e.d.

Remark 5.2 A careful inspection of the proof of Lemma 5.1 shows that the core on $\Gamma^{c h}$ satisfies $\mathrm{RGP}^{c c}$ and $\mathrm{CRGP}^{c c}$ as well.

Theorem 5.3 The bounded core on $\Gamma^{c h}$ is the unique solution that satisfies ZIG, COV, WRGP ${ }^{c c}$, $\mathrm{CRGP}^{c c}$, and BOUND.

Proof: By Lemma 4.3 and Lemma 5.1 the bounded core satisfies the desired axioms. In order to verify uniqueness, let $\sigma$ be a solution on $\Gamma^{c h}$ that satisfies ZIG, COV, WRGP ${ }^{c c}$, CRGP $^{c c}$, and BOUND. By Lemma $5.4, \sigma$ is a subsolution of the core. Let $(N, \preceq, v) \in \Gamma^{c h}$. If $|N| \leqslant 2$, then ZIG, COV, and BOUND imply that $\sigma(N, \preceq, v)=C^{b}(N, \preceq, v)$ (see proof of Theorem 4.4). Now, let $|N|>2$ and $x \in \sigma(N, \preceq, v)$. As $\sigma$ coincides with $C^{b}$ for any 2-person game in $\Gamma^{c h}$, PO of $\sigma$ and $\operatorname{CRGP}^{c c}$ of $C^{b}$ imply $x \in C^{b}(N, \preceq, v)$. Hence, $\sigma(N, \preceq, v) \subseteq C^{b}(N, \preceq, v)$. The opposite inclusion is shown by interchanging the roles of $\sigma$ and $C^{b}$.

q.e.d.

Note that the foregoing proof is similar to the proof of Peleg's (1986) axiomatization of the prekernel.

Lemma 5.4 If $\sigma$ is a solution on $\Gamma^{c h}$ that satisfies $\mathrm{COV}$, $\mathrm{WRGP}^{c c}$, and $\mathrm{BOUND}^{s}$, then $\sigma$ is a subsolution of the core.

Proof: Let $(N, \preceq, v) \in \Gamma^{c h}$. If $|N| \leqslant 2$, the proof is finished by COV and BOUND ${ }^{s}$. If $|N| \geqslant 3$, then by WRGP ${ }^{c c}$ applied to one-person reduced games (note that any singleton coalition is connected), any element of $\sigma(N, \preceq, v)$ is Pareto optimal. Thus, $\sigma$ satisfies PO. Let $x \in \sigma(N, \preceq, v)$. As the core satisfies $\mathrm{CRGP}^{c c}$ by Remark $5.2, x \in C(N, \preceq, v)$.

q.e.d.

Corollary 5.5 The core is the maximum solution on $\Gamma^{c h}$ that satisfies $\mathrm{COV}$, WRGP ${ }^{c c}$, and $\mathrm{BOUND}^{s}$.

In order to show that each of the five axioms in Theorem 5.3 is logically independent of the remaining axioms, provided that $|U| \geqslant 3$, we define five solutions $\sigma^{i}, i=1, \ldots, 5$, so that $\sigma^{i}$ exclusively violates the 
$i$-th axiom. For $(N, \preceq, v) \in \Gamma^{c h}$ define

$$
\begin{aligned}
\sigma^{1}(N, \preceq, v) & =\emptyset \text { (the "empty" solution), } \\
\sigma^{2}(N, \preceq, v) & =\{z\}, \text { where } z_{i}=\frac{v(N)}{|N|} \text { for all } i \in N \text { (the "equal split" solution), } \\
\sigma^{3}(N, \preceq, v) & =\left\{x \in X(N, \preceq, v) \mid x_{i} \geqslant b_{i}(N, \preceq, v) \text { for all } i \in N\right\} \text { (the "reasonable below set"), } \\
\sigma^{4}(N, \preceq, v) & =\operatorname{ext}(C(N, \preceq, v)), \text { and } \\
\sigma^{5}(N, \preceq, v) & =C(N, \preceq, v) .
\end{aligned}
$$

Clearly, $\sigma^{1}$ and $\sigma^{2}$ exclusively violate ZIG (if $|U| \geqslant 1$ ) and $\operatorname{COV}$ (if $|U| \geqslant 2$ ), respectively. By Remark 5.2 the core satisfies $\mathrm{RGP}^{c c}$ and $\mathrm{CRGP}^{c c}$. Moreover, the bounded core is a subsolution of the core, and the core is unbounded for any two-person game with a connected hierarchy. Thus, $\sigma^{5}$ exclusively violates BOUND (if $|U| \geqslant 2$ ).

Example 5.6 below shows that neither $\sigma^{3}$ nor $\sigma^{4}$ coincides with the bounded core provided that $|U| \geqslant 3$.

The bounded core of a game with a connected hierarchy and two persons coincides with the unique extreme point of the core of the game. Hence $\sigma^{3}$ and $\sigma^{4}$ coincide with the bounded core for all games in $\Gamma^{c h}$ with at most two players. In general the bounded core is a subsolution of $\sigma^{3}$ by Lemma 3.3 and a supersolution of $\sigma^{4}$ by definition. Clearly, $\sigma^{3}$ and $\sigma^{4}$ satisfy COV so that they exclusively violate WRGP $^{c c}$ and $\operatorname{CRGP}^{c c}$ (if $|U| \geqslant 3$ ), respectively.

Example 5.6 Let $(N, \preceq, v)$ be defined by $N=\{1,2,3\}, 1,2 \prec 3$, and $v(\{1,2\})=v(N)=1, v(S)=0$ for all $S \in\{\emptyset,\{1\},\{2\}\}$. Then $b_{1}(v)=b_{2}(v)=b_{3}(v)=0$ so that $(0,0,1) \in \sigma^{3}(N, \preceq, v) \backslash C(N, \preceq, v)$. Moreover, $\left(\frac{1}{2}, \frac{1}{2}, 0\right) \in C^{b}(N, \preceq, v) \backslash \operatorname{ext}(C(N, \preceq, v))$ because $(1,0,0),(0,1,0) \in C(N, \preceq, v)$.

\section{References}

Bondareva, O. N. (1963): "Some applications of linear programming methods to the theory of cooperative games," Problemi Kibernitiki, 10, 119-139.

Derks, J. J. M., And R. P. GiLles (1995): "Hierarchical organization structures and constraints on coalition formation," International Journal of Game Theory, 24, 147 - 163.

FAigle, U. (1989): "Cores of games with restricted cooperation," Zeitschrift für Operations ResearchMathematical Methods of Operations Research, 33, 405 - 422.

FAigle, U., AND W. KERN (1992): "The Shapley value for cooperative games under precedence constrainrs," International Journal of Game Theory, 21, 249 - 266.

Fujishige, S. (2005): Submodular functions and optimization, vol. 58 of Annals of Discrete Mathematics. Elsevier, Amsterdam, 2nd edn.

Grabisch, M. (2011): "Ensuring the boundedness of the core of games with restricted cooperation," Annals of Operations Research, 191, 137154.

Hwang, Y.-A., AND P. Sudhölter (2001): "Axiomatizations of the core on the universal domain and other natural domains," International Journal of Game Theory, 29, 597 - 623. 
Maschler, M., B. Peleg, and L. S. Shapley (1972): "The kernel and bargaining set for convex games," International Journal of Game Theory, 1, 73-93.

Peleg, B. (1986): "On the reduced game property and its converse," International Journal of Game Theory, 15, $187-200$.

Peleg, B., And P. Sudhölter (2007): Introduction to the Theory of Cooperative Games, Theory and Decisions Library, Series C: Game Theory, Mathematical Programming and Operations Research. Springer-Verlag, Berlin, Heidelberg, 2nd edn.

Rockafellar, R. T. (1970): Convex Analysis. Princeton University Press, New Jersey.

Shapley, L. S. (1967): "On balanced sets and cores," Naval Research Logistics Quarterly, 14, 453 460.

Tomizawa, N. (1983): "Theory of hyperspace (XVI) — on the structure of hedrons," Papers of the Technical Group on Circuits and Systems CAS82-172, Inst. of Electronics and Communications Engineers of Japan, In Japanese. 